Berufsbilder bzw. das Selbstverständnis von Berufsgruppen sind keine isolierten, vom gesellschaftlichen Wandel und technischen Entwicklungen abgekoppelten Entitäten. Dies zeigt sich in der jüngeren Vergangenheit gerade in der Medizin deutlich: Die Diskussion über Aufgaben, Ziele und Prioritätensetzung sowie das Rollenverständnis der involvierten Akteure wird in diesem Bereich in den letzten Jahren intensiv geführt. Aktuelle Beispiele sind der unlängst veröffentlichte Bericht der Schweizerischen Akademie der Medizinischen Wissenschaften SAMW über «Die zukünftigen Berufsbilder von Ärztinnen/Ärzten und Pflegenden in der ambulanten klinischen Praxis» (SÄZ Nr. 46/2007) oder die ebenfalls von der SAMW initiierte Tagung zum Thema «Human Enhancement» vom vergangenen 21. November in Bern. Berufsethische Fragen grundsätzlicher und spezieller Art, die durch die Entwicklungen und Tendenzen in der modernen Medizin aufgeworfen werden, sollen in der Schweizerischen Ärztezeitung in ihrer ganzen Breite diskutiert werden können. Giovanni Maio vertritt im folgenden Artikel einen pointiert konservativen, also bewahrenden Standpunkt: Ärztinnen und Ärzte sollten trotz der zunehmenden Ökonomisierung und Entpersonalisierung nicht zu reinen Dienstleistern werden, sondern karitative Helfer bleiben.

Die Redaktion

\title{
Die moderne Dienstleistungsmedizin auf ethischem Prüfstand
}

Giovanni Maio

Interessenkonflikt: Es besteht kein Interessenkonflikt. Der korrespondierende Autor versichert, dass keine Verbindungen mit einer Firma, deren Produkt in dem Artikel genannt ist, oder einer Firma, die ein Konkurrenzprodukt vertreibt, bestehen. Die Präsentation des Themas ist unabhängig und die Darstellung der Inhalte produktneutral.

Korrespondenz:

Prof. Dr. Giovanni Maio Lehrstuhl für Bioethik

Interdisziplinäres Ethik-Zentrum Freiburg

Stefan-Meier-Strasse 26

D-79104 Freiburg i.Br.

Tel. +497612035034

Fax +497612035032

maio@egm.uni-freiburg.de

\section{Einleitung}

Obwohl die Medizin eine soziale Institution ist, die sich von ihrer Grundidentität her der Hilfe für in Not geratene kranke Menschen verschreibt, kommt der Begriff der ärztliche Hilfe in den gegenwärtigen öffentlichen Diskussionen um die moderne Medizin so gut wie gar nicht vor. Viel häufiger ist heute von der ärztlichen Dienstleistung die Rede, von Qualitätssicherung, von Wettbewerbsfähigkeit, von Ansprüchen, von Rechten. Fast scheint es, als sei in den heutigen Gesundheitsstrukturen, die immer mehr mit marktwirtschaftlichen Termini überzogen werden, jener Arzt, der sich aus einer karitativen Grundhaltung heraus der Hilfe für in Not geratene Menschen verpflichtet fühlt, eine antike und fremde Figur geworden. Die Medizin durchläuft gegenwärtig einen grundlegenden Transformationsprozess mit enormen Auswirkungen auf die Arzt-Patienten-Beziehung. Am Ende einer solchen Transformation wird die klassische, an der Fürsorge orientierte ArztPatienten-Beziehung durch eine an den Kundenwünschen orientierte Dienstleister-KundenBeziehung ersetzt. Wie ist ein solcher Umformungsprozess der Medizin in ethischer Hinsicht zu bewerten? Um als Disziplin der Reflexion ihre Glaubwürdigkeit zu wahren, hat die Medizinethik die Aufgabe, nicht nur Technikfolgenabschätzung zu betreiben, sondern es kommt ihr gleichsam die Aufgabe zu, die Entwicklung

\section{La médecine entrepreneuriale} moderne au banc d'essai

\section{de l'éthique}

La médecine moderne se transforme rapidement. Cette transformation concerne avant tout la relation entre le médecin et son patient. Le patient se comprend souvent plutôt comme un consommateur que comme un malade. Comme consommateur il exige de la médecine l'abolition de chaque souffrance de la vie quotidienne. Le médecin moderne est en train d'éradiquer son identité caritative et de la remplacer par une identité de technicien et d'entrepreneur impersonnel. L'article analyse les implications éthiques de cette transformation. La médecine moderne est critiquée par son manquement de réflexion en tant que les désirs des consommateurs médicaux sont souvent une manifestation d'un narcissisme moderne qui est soutenu et renforcé par une médecine du désir. L'article fait la proposition de comprendre la médecine comme une institution sociale qui est basée sur un caractère de confiance et de bienfaisance. Cette base est un fondement éthique qui ne peut pas être aboli sans abolir la médecine elle-même. 
der modernen Medizin grundlegend zu hinterfragen. Eine solche kritische Beleuchtung der Paradigmen der modernen Medizin soll im folgenden versucht werden. Die Transformation der Medizin hin zu einem Dienstleistungsbetrieb auf Wunsch setzt, sowohl was das Patientenbild als auch was das Arztbild angeht, ein bestimmtes Verständnis voraus. Beide Selbstverständnisse sollen im folgenden kritisch beleuchtet werden, um in einem letzten Teil einen Vorschlag für eine tragfähige Medizin der $\mathrm{Zu}$ kunft zu machen.

\section{Der moderne Patient als Konsument in einer Vertragsbeziehung}

Im Zuge des gegenwärtigen Wandels der modernen Medizin wird nicht zuletzt der Patient zunehmend umdefiniert, indem er immer weniger als Patient im Sinne eines notleidenden Mitmenschen gesehen wird. Statt dessen wird im Patienten immer mehr der mögliche Verbraucher von medizinischen Dienstleistungen entdeckt [1]. Der notleidende Patient, der sich hilfesuchend an seinen Arzt gewendet hat, wird zunehmend ersetzt durch den begehrenden und fordernden Patienten, der nicht nach Hilfe sucht, sondern der als wohlinformierter Bürger und Beitragszahler seine Rechte und Ansprüche einlösen zu müssen glaubt. Was heute zwischen Arzt und Patient verhandelt wird, ist in dieser neuen Partnerbeziehung kein wertvolles, unverzichtbares und einzigartiges Hilfsangebot des einen speziellen Arztes, sondern es ist eine beliebig austauschbare und von jedwedem Dienstleisterarzt in gleicher Weise anzubietende Gesundheitsdienstleistung geworden, die auf ihre abprüfbaren Qualitätskriterien erst abgeklopft werden muss, bevor sie in Anspruch genommen wird. An die Stelle einer personalen Beziehung zwischen einem Notleidenden und einem Helfer ist eine rein sachliche Vertragsbeziehung zwischen einem Dienstleistungsanbieter und einem Dienstleistungskonsumenten getreten. Der moderne Patient ähnelt immer mehr einem Verbraucher, der nicht mit einem Hilfsbegehren, sondern mit Qualitätsansprüchen kommt und der dem Kunden gleich selbstverständlich als König behandelt werden möchte und nur das Beste zum geringsten Preis verlangt. Schlussendlich stellt dieser Wandel des ArztPatienten-Verhältnisses nichts weniger als einen Wandel von einem Vertrauens- zu einem Vertragsverhältnis dar. Die Arzt-Patienten-Beziehung unterliegt damit einer zunehmenden Versachlichung, Verrechtlichung und Entpersonalisierung.

\section{Der moderne Arzt als unparteiischer Wunscherfüller}

Dem modernen Patienten als Konsumenten steht der moderne Arzt als Dienstleistender gegenüber. Auch der moderne Arzt richtet sich darauf ein, nicht mehr nur Leiden zu lindern und in Not geratene Menschen mit einer anteilnehmenden Hilfe zu begegnen. Statt dessen verwandelt sich der moderne Arzt zunehmend zu einem Anbieter von Gesundheitsleistungen, der mit seinem Wissen und Können nicht mehr zugleich auch seine Person in den Dienst der Hilfe für in Not geratene Menschen stellt, sondern der nunmehr lediglich sein Wissen und seine Technik seinen Nichtpatienten unverbindlich anbietet, ohne diese Wünsche weiter zu hinterfragen. An die Stelle einer personalen Fürsorgebeziehung tritt eine zweckrationale Dienstleistungsbeziehung, über die nicht nur Krankheitsbehandlungen vollzogen, sondern zugleich auch persönliche Wünsche und Vorlieben erfüllt werden.

Ob Anti-Aging-Produkte, kosmetische Massnahmen, Brustimplantate oder Sectio auf Wunsch, ob Sexualhormone gegen das Altern oder Mittel zur Abschaffung des «beschwerlichen» Menstruationszyklus, ob Ritalin für nichtkranke Kinder, ob stimmungsaufhellende Medikamente für nichtkranke Patienten, ob IGEL-Leistungen jeglicher Art - in vielen Bereichen der modernen Medizin hat die wunscherfüllende Medizin von Dienstleisterärzten bereits breiten Eingang gefunden [2, 3]. Der moderne Mensch möchte jegliche Behinderung, jegliche Beeinträchtigung beseitigt wissen, und er wird keine Mühe haben, Ärzte zu finden, die ihm genau dies versprechen - alles nach Wunsch und Belieben.

Was der moderne Arzt anbietet, ist im Kontext einer solchen wunscherfüllenden Medizin kein persönlicher und unverwechselbarer Dienst am Menschen, sondern es ist die Lieferung eines Produktes, bei dem es lediglich um die «Einwandfreiheit» des Produktes selbst und die Garantie des Funktionierens dieses Produktes geht. Ob die mit der Verwendung des Produktes verfolgte Zielsetzung eine gute und vertretbare ist, spielt somit in einem solchen Dienstleistungsdenken keine zentrale Rolle. Der Arzt bietet nicht mehr an als eine Sachleistung, die im Rahmen eines Vertrages «abgewickelt» wird. Solange der Dienstleisterarzt hier ausreichend über die Risiken informiert hat, hat er - nach dieser Auffassung - seiner «moralischen» Verpflichtung ausreichend Genüge getan und kann sich vermeintlich jeglicher weiteren moralischen Verantwortung entledigt fühlen. Trägt der Dienstleisterarzt tatsächlich keine weitere Verantwortung als die der ausreichenden Information? 


\section{Ethische Kritik}

\section{der Dienstleistungsmedizin auf Wunsch}

\section{Die Dienstleistungsmedizin als Bestärkerin einer Anspruchsmentalität}

Der moderne Mensch ist ein Anspruchsmensch, der nicht bereit ist, das (vermeintlich) Unvollkommene anzunehmen, sei es das Unvollkommene des Gegenübers, das er herabwürdigt, sei es das Unvollkommene an sich selbst, das er mit allen Mitteln unablässig abzustreifen versucht. Der moderne Mensch ist unfähig geworden, hinter jeder vermeintlichen Unvollkommenheit nur die äussere Manifestation eines an sich wertvollen Lebens zu erblicken. Genau mit dieser Ideologie sind Ärzte konfrontiert, wenn sie es mit Menschen zu tun haben, die von ihnen verlangen, dass sie ihren Körper, ganz gleich wie alt dieser ist, schnellstmöglich reparieren, und die kein Verständnis dafür haben, dass der Mensch in seinem Leibsein stets angewiesen ist auf seinen Körper und dass allein seine Leibhaftigkeit es dem Menschen verunmöglicht, absolut frei von Beschwerden und körperlichen Nöten zu sein. Der moderne Mensch empfindet sich tagtäglich als Leidender und ruft daher die Medizin an, aber die Ursache des Leides ist in zunehmendem Masse nicht das Beschwerdebild selbst, sondern die Haltung des Anspruchs auf ein «mangelloses Leben» [4]; der moderne Mensch leidet an seiner utopischen Erwartung. Der Wunsch eines jeden Menschen nach Beschwerdefreiheit ist nachvollziehbar und legitim; das Problem liegt daher nicht am Wunsch nach Beschwerdefreiheit, sondern einzig und allein an der Verabsolutierung dieses Wunsches im Zuge der Überhöhung der «Gesundheit». Von diesem Moment an, da die Beschwerdefreiheit zur absoluten und unverzichtbaren Voraussetzung für die Wertschätzung des Lebens gemacht wird, von da an wird der ursprünglich legitime Wunsch schliesslich zur Obsession und damit zur lebensverneinenden Ideologie. Im Zuge einer Anspruchsmentalität wird der Verlust der Gesundheit gleichgesetzt mit Verlust der Möglichkeit, ein gutes Leben zu führen. Dass die moderne Medizin sich unreflektiert einer solchen Ideologie weitestgehend angeschlossen hat, ohne ihr eine andere Vorstellung von Menschsein und von Sinnerfüllung entgegenzustellen, ist daher ein verhängnisvolles Versäumnis.

Zunächst scheint die Medizin wehrlos dem Anspruchsverhalten vieler Patienten ausgeliefert zu sein. Doch eine solche Sichtweise lässt ausser acht, dass die Ansprüche der Patienten letzten Endes von der Medizin selbst genährt werden. Dies gilt nicht nur für die wunscherfüllende äs- thetische Medizin, die sich mit ihren Angeboten stellenweise zu einem Zahnrad in der Beautyindustrie macht. Dies gilt auch für den expandierenden Bereich der Anti-Aging-«Medizin». Aber auch für den Lebensanfang trifft dieser Zusammenhang zwischen Erwartungshaltung und Angeboten der Medizin zu. So ist es beispielsweise nicht die schwangere Frau gewesen, die nach der Präimplantationsdiagnostik gefragt hat, sondern es ist die Medizin selbst gewesen, die auf die Idee der vorgezogenen genetischen Diagnostik im Embryonalstadium gekommen ist. Die Medizin selbst hat diese Technik erfunden und sie den werdenden Müttern als die beste Lösung ihrer Angst vor einem behinderten Kind offeriert. Die Medizin selbst hat auch die Pränataldiagnostik erfunden und sie allen werdenden Müttern als «Segen» für das «Problem Schwangerschaft» angeboten. Damit hat die Medizin selbst die Schwangerschaft zu einem medizinischen «Problem» gemacht, nachdem sie über Jahrhunderte hinweg als ein Zustand der «guten Hoffnung» gegolten hatte.

Die moderne Medizin ist wiederum selbst auf die Idee gekommen, das ungeborene Kind nicht «nur» mit dem Ultraschall, sondern zwischenzeitig sogar mit der Magnetresonanztomographie durchzumustern. Zwar steht am Anfang der Indikation zur Magnetresonanztomographie im Mutterleib - wie auch ursprünglich zur Pränataldiagnostik - der Wunsch der Medizin, besonders gefährdetes Leben besser und damit auch sinnvoller zu kontrollieren, aber es ist befremdend, wie sorglos und unreflektiert diese diagnostische Erweiterung von vielen Ärzten angepriesen wird. So ist von der Logik des modernen Patienten als anspruchsvollen Konsumenten her abzusehen, dass diese Grundentscheidung der Medizin, das vorgeburtliche Leben auf eine so minutiöse Weise sichtbar zu machen, vielfältige Ansprüche von vielen Eltern evozieren wird.

\section{Die Dienstleistungsmedizin als Bestärkerin des modernen Narzissmus}

Diese unreflektierte Grundhaltung vieler moderner Ärzte - in Kombination mit einer ökonomischen Identität der modernen Medizin - hat zur Folge, dass die moderne Medizin ihre Nochnicht-Patienten gerade nicht mit dem problematischen Charakter ihrer Erwartungen und mit dem oftmals narzisstischen Gehalt ihrer Wünsche an die Medizin konfrontiert. Statt dessen reagiert die moderne Medizin damit, die von Selbstbefangenheit geprägten Ansprüche der Patienten, soweit es irgend geht und mit allen zur Verfügung stehenden Mitteln, zu erfüllen. In der Konfrontation mit vielen Menschen, die sich 
in irrationaler Weise an der Utopie des mangellosen Lebens und an der Ideologie der Leistungsfähigkeit orientieren und damit ideologisch gefärbte Erwartungen an das eigene Leben und auch an das Leben der eigenen Nachkommen stellen, hat die moderne Dienstleistungsmedizin - in weiten Teilen - keine andere Antwort gefunden, als sich diese Erwartungen für die Steigerung des eigenen Profits zunutze zu machen [5].

Nur zuwenig wird bedacht, dass ab dem Moment, da der Dienstleisterarzt Sachleistungen anbietet und sogar mehr oder weniger direkt Werbung dafür betreibt, er sich mitverantwortlich macht für die Folgen, die aus der Anwendung «seiner» Techniken resultieren. So ist der moderne Dienstleisterarzt und Wunscherfüller dafür mitverantwortlich, dass vorgeburtliches Leben als kontrollierbare «Sachleistung» der technischen Medizin betrachtet wird, er ist dafür mitverantwortlich, dass an dieses werdende Leben Bedingungen gestellt werden, dass es nicht mehr bedingungslos angenommen wird. Der moderne Dienstleisterarzt ist dafür mitverantwortlich, dass junge (wie alte) Menschen glauben, ihre Körperform verändern zu müssen, um Anerkennung bei ihren Gleichaltrigen (Jungen wie Alten) zu finden. Ich spreche hier bewusst von «mit»-verantwortlich, wohl wissend, dass beim Zustandekommen solcher Wünsche an die Medizin soziale Erwartungen eine grosse Rolle spielen, aber die Medizin trägt die Verantwortung dafür, dass die sozialen Erwartungen zu spezifischen Wünschen führen, die nicht von ungefähr medizinische Wünsche sind, weil die reine Dienstleistungsmedizin gegen Geld medizinische Massnahmen als vermeintliche Lösung für soziale Probleme anbietet. Daher ist der moderne Dienstleisterarzt mitverantwortlich für eine moderne Leistungsgesellschaft, die nur jenen Menschen Zugang verschafft, die den Kriterien der Leistungsfähigkeit genügen. Eine solche Medizin hat sich dafür entschieden, keine Institution der Hilfe mehr zu sein, sondern nur noch Erfüllungsgehilfin einer rastlosen Gesellschaft, die so etwas wie Sinn nur noch in der wahnhaften Selbstgestaltung erblickt [6]. Diese Komplizenschaft der modernen Medizin mit den Ideologien der modernen Leistungs- und Konsumgesellschaft ist um so tragischer, als die äussere Erscheinung oder die Leistungsfähigkeit für so viele moderne Menschen gerade deswegen einen so hohen Stellenwert einnehmen, weil viele Menschen in einem Sinndefizit leben, das sie mit solchen Äusserlichkeiten zu kompensieren versuchen [7]. Angesichts eines solchen Sinndefizits kann die Aufgabe der Medizin als Institution der Hilfe nicht auf eine sachliche und unre- flektierte Dienstleistung nach Wunsch reduziert werden. Eine solche Dienstleistungsmedizin auf Wunsch wird dem medizinischen Anspruch, einen Dienst am Menschen zu erfüllen, nicht gerecht, weil diese Medizin den Menschen nur in seiner selbstbefangenen Begehrlichkeit wahrnimmt und sich gerade nicht mit dem Gegenüber in seiner gesamten Existenz beschäftigt. Würde der moderne Arzt dies nämlich tun, müsste er sich viel mehr dafür interessieren, warum diese Menschen gerade mit solchen Begehren ihn als Arzt aufsuchen. Statt diese Menschen mit dem utopischen und zugleich narzisstischen Gehalt ihrer Ansprüche zu konfrontieren, bestätigt das moderne ärztliche Handeln die Gesundheitskonsumenten in ihrer Selbstbefangenheit und Egozentrik. Daher geht die ethische Verantwortung des modernen Dienstleisterarztes bei weitem über die Verantwortung zur umfassenden Aufklärung der Risiken hinaus. Dies lässt sich gerade am Umgang der modernen Medizin mit den Ansprüchen des alternden Menschen verdeutlichen.

\section{Die Dienstleistungsmedizin als Verstärkerin einer altersfeindlichen Grundhaltung}

Viele Menschen suchen den «Arzt» auf, damit dieser ihnen helfe, die physiologischen Alterserscheinungen $\mathrm{zu}$ kaschieren. Hierfür hat die moderne Medizin ein breites Arsenal an wirksamen Methoden entwickelt, die in allen Handbüchern (für kosmetische Dermatologie, kosmetische Chirurgie, Anti-Aging-Medizin usw.) aufgeführt werden. Die Tatsache, dass die Medizin das Altsein mit technischen Mitteln zu verbergen versucht und dass sie hierfür Mittel nicht nur anwendet, sondern auch anpreist, ist alles andere als trivial. Denn damit suggeriert die Medizin, dass das Altaussehen - und damit das Altsein ein bedauernswürdiger Zustand ist, der behoben werden müsste. Im Zuge einer unreflektierten Dienstleistungsmedizin auf Wunsch verstärkt die Medizin die modernen Tendenzen, das Altsein als etwas Defizitäres zu betrachten. Eine so pejorisierende Betrachtung des Altseins ist zwar kein Spezifikum des 21. Jahrhunderts, vielmehr hat es seit der Antike immer wieder solche Assoziationen gegeben [8]. Die moderne Medizin macht sich den wiederbelebten Trend zur Kultivierung der Jugendlichkeit allerdings in einer exzessiven Weise zunutze und schlägt daraus Profit. Dies ist insofern problematisch, als der Profit nur durch eine fragwürdige und kritikwürdige Vorstellung vom Menschsein möglich geworden ist.

Indem die Medizin mehr oder weniger unreflektiert Mittel anpreist, durch die die Älteren 
nach jugendlichen Vorbildern äusserlich umgestaltet werden sollen, macht sich die Medizin zur Komplizin einer Gesellschaft, die im Altsein nur noch eine Schwundstufe des Menschen sieht. Die Medizin sorgt auf diese Weise mit dafür, dass das Altsein nur dann akzeptiert wird, wenn der Mensch auch im Altsein noch jugendlich «schön» ist. Versteht man die Medizin als eine Institution der Hilfe, so könnte man ihr im Umgang mit dem alternden Menschen eine andere helfende Aufgabe zuschreiben als jene, dem alternden Menschen dabei zu helfen, das Altsein und die spezifischen Merkmale des Altwerdens $\mathrm{zu}$ ignorieren und zu verdrängen. Indem ältere Menschen von der Medizin äusserlich jugendlich gemacht werden, werden diese alten Menschen gerade nicht in ihrem Altsein respektiert, sondern nur insofern sie noch so geblieben sind wie die Menschen in der mittleren Lebensphase. Mit allen Mitteln nicht alt aussehen zu wollen ist somit Ausdruck einer Ideologisierung der mittleren Lebensphase. Eine solche Selbstdeutung des Menschen ist in ethischer Hinsicht problematisch, weil sie den Wert und den Sinn des Altseins ignoriert.

Als Institution der Hilfe könnte die Medizin sich dafür einsetzen, einen gesunden Umgang mit dem Altwerden zu unterstützen. Sie könnte alternativ zur unreflektierten Wunscherfüllung den Altgewordenen aufzeigen, dass man auch und gerade in seiner äusseren wie inneren Gebrechlichkeit ein lebenswertes Leben führen kann, sofern man es schafft, sich von den Ideologien der Jugendlichkeit und Leistungsorientiertheit der mittleren Lebensphase zu lösen. Als Institution der Hilfe könnte die Medizin um die Einsicht werben, dass das Gute nicht allein durch das gute Machen und Gestalten verwirklicht werden kann, sondern dass gerade im Altern - und nicht nur da - das Gute auch durch ein Sein zur Realisierung kommen kann. Kaum eine andere Domäne unserer Gesellschaft nimmt einen so starken Einfluss auf unsere Bilder vom Altsein wie die Medizin. Daher ist es eine moralische Verpflichtung der Medizin, über ihr Tun gerade im Zuge einer zunehmenden Dienstleistungsmentalität kritischer nachzudenken.

\section{Zusammenfassung und Schlussfolgerungen}

Zusammenfassend lässt sich kritisch festhalten, dass die moderne Medizin nicht nur Wünsche erfüllt, sondern auch neue Wünsche weckt und damit die Menschen in ihren zum Teil irrationalen und egozentrischen Ansprüchen weiter stärkt. Was durch eine am Leitbild des Kunden orientierte und moralisch unreflektierte Medizin geschieht, ist eine Lenkung der Bedürfnisse, eine Weckung von Wünschen, deren Inhalte allein nach der technischen Machbarkeit, der individuellen Wünschbarkeit und der privaten Finanzierbarkeit ausgerichtet werden. Versteht man die Medizin als eine soziale Institution, so wird deutlich, dass durch diese Beliebigkeit der Wunscherfüllung der «soziale» Charakter der Medizin vollkommen aus dem Blick gerät.

Für den modernen Arzt ist es angesichts der dargelegten Entwicklungen eine besondere Herausforderung, sich nicht zu einem reinen Dienstleistenden auf Wunsch degradieren zu lassen und nicht zum Spielball einer verkürzt verstandenen Patientenautonomie zu werden. Diese Herausforderung ist für den modernen Arzt besonders schwierig zu bestehen, weil die modernen Krankenhausstrukturen so aufgebaut sind, dass der moderne Arzt finanziell dann belohnt wird, wenn er Dienstleister ist, und Nachteile befürchten muss, wenn er im wahren Sinne als Arzt handelt. Die Anreizsysteme der modernen Kliniken, die sich zunehmend als Unternehmen und nicht mehr als karitative Stätten verstehen, sind derart, dass der Arzt, der sich als Person einbringt und einen nicht nur sachlich richtigen, sondern vor allem einen humanen Dienst verrichten will, zunehmend zum Fremdling im neuen Krankenhauskonzept wird. Der humane Dienst, der einst identitätsstiftend für die - aus dem Christentum stammenden - Krankenhäuser war, wird im Zuge der neuen marktwirtschaftlichen Entwicklung der Kliniken zunehmend zur idealistischen Beigabe, ist aber längst nicht mehr das identitätsstiftende Element der modernen Kliniken. Damit degenerieren die modernen Kliniken zu unpersönlichen Fliessbandbetrieben, zu Reparaturfabriken ohne Seele. Wenn die Medizin als Disziplin der Hilfe für in Not geratene Menschen und damit als Ort des Vertrauens überleben will, so wird sie gut beraten sein, sich auf ihre Kernkompetenzen zurückzubesinnen. Die zentrale Befähigung des Arztes besteht darin, ein verstehender und einfühlender Helfer für in Not geratene Menschen zu sein. Im Mittelpunkt einer solchen Kernkompetenz darf daher nicht die Technik, nicht die Machbarkeit alleine stehen und erst recht nicht die Profitabilität des Spitals, sondern im Mittelpunkt muss das Ziel stehen, einen Dienst am Mitmenschen zu vollbringen. Für dieses Ziel braucht der Arzt nicht nur Wissen und Fertigkeiten, sondern er braucht durch alle diese Qualifikationen hindurch eine moralische Grundhaltung, die in jedem Menschen, mag er noch so krank, noch so klein, noch so unheilbar sein, eine einzigartige und unschätzbare Person erblickt, die selbst 
dann, wenn sie von allen anderen verlassen worden ist, mit Recht darauf vertrauen darf, zumindest in ihrem Arzt ein verstehendes und helfendes Gegenüber zu finden. Wenn die moderne Medizin diese vertrauensvolle Helferperson Arzt immer weiter ersetzt durch einen sachlichen Dienstleister in einem Gesundheitsmarkt, wird sie zwar den gesunden Dienstleistungsverbrauchern mehr Freiheit schenken, aber um den Preis, dass die kranken Menschen nicht mehr wissen werden, ob sie sich auch in kranken und schweren Tagen einem solchen Dienstleister-«Arzt» blind anvertrauen können. Daher erscheint die Doppelrolle des modernen Arztes, einerseits Dienstleistungsanbieter und -verkäufer und andererseits Helfer für in Not geratene Menschen zu sein, kaum in einer Person vereinbar. Da der Dienstleisterarzt für Gesunde sehr viel lukrativere Geschäfte wird machen können - allein weil die Klientel der Gesunden grösser ist als das Kollektiv der Kranken -, steht zu befürchten, dass im Zuge einer fortschreitenden Ökonomisierung der Medizin die Helferperson Arzt sukzessive verdrängt werden wird. Als Grundform der menschlichen Praxis bleibt die Zuwendung eines ärztlichen Helfers ein Bestandteil der gesamten Gesellschaft, auf den kein vernünftiger Mensch zu verzichten bereit sein dürfte. Daher wäre die Ablösung einer solchen sittlichen Helferperson durch einen dem Gesundheitskult dienenden wunscherfüllenden Dienstleister für die gesamte Gesellschaft ein nicht ersetzbarer Verlust. Im Interesse aller kranken Menschen wird die moderne Medizin somit nicht umhinkommen, darüber nachzudenken, wie sie trotz der zunehmenden Ökonomisierung und Entpersonalisierung genau diesen fast schon verlorengegangenen Arzt als karitativen Helfer bewahren kann.

\section{Literatur}

1 Lanzerath D. Krankheitsbegriff und Zielsetzungen der modernen Medizin: vom Heilungsauftrag zur Antiaging-Dienstleistung? Gesundheit und Gesellschaft. 2003;3(3):14-20.

2 Kettner M. «Wunscherfüllende Medizin» zwischen Kommerz und Patientendienlichkeit. Ethik in der Medizin. 2006;1(18):81-91.

3 Maio G. Die Präferenzorientierung der modernen Medizin als ethisches Problem. Ein Aufriss, am Beispiel der Anti-Aging-Medizin. Zeitschrift für medizinische Ethik. 2006;52:339-54.

4 Kamlah W. Philosophische Anthropologie: Sprachkritische Grundlegung und Ethik. Mannheim: Bibliographisches Institut; 1973.

5 Maio G. Medizin im Umbruch. Ethisch-anthropologische Grundfragen zu den Paradigmen der modernen Medizin. Zeitschrift für medizinische Ethik. 2007;53:229-54.

6 Maio G. Ist die ästhetische Chirurgie überhaupt noch Medizin? Eine ethische Kritik. Handchir Mikrochir Plast Chir. 2007;39:189-94.

7 Marquard O. Zur Diätetik der Sinnerwartung. Philosophische Bemerkungen. In: Marquard O. Apologie des Zufälligen. Stuttgart: Philosophische Studien; 1986. S. 33-53.

8 Engelhardt D. Der Gesundheitsbegriff im Wandel der Geschichte. Widerspruch. 2004;42:25-36. 